\title{
STRUCTURE PERFECTION STUDY OF CRYSTALS CONTAINING MICRO- AND MACRODISTORTIONS BY X-RAY ACOUSTIC METHOD
}

\author{
V.I. Kintupa, D.O. Grigoryev and A.Ya. Dzyublik \\ Institute of Semiconductor Physics, National Academy of Sciences \\ Prospekt Nauki 45, 252028 Kiev-28, Ukraine
}

\begin{abstract}
The X-ray acoustic method for determination the structure perfection integral characteristics is suggested for slightly imperfect dislocation-free crystals. The method is suitable for investigation of a crystal disturbed both by localized (microdefects) and by distributed (macrostrains) structure defects. It is based on the analysis of dependence of the distance $\Delta x$ between two minima, arising in the spatial intensity profile $I(x)$ of the X-ray beam diffracted by acoustically excited crystal, upon ultrasound frequency $\nu_{\mathbf{s}}$. Using the data $\Delta x\left(\nu_{\mathrm{s}}\right)$ for two selected reflections, we calculated the values of the extinction lengtlis $\Lambda$ which enabled us to identify the predominate type of structure disturbances as well as to estimate the static Debye-Waller factors $\mathrm{e}^{-L}$ and the period of the main macrodeformation $\lambda_{M}$ for a sample containing simultaneously microdefects and periodic long range deformations. Such approach was used for studying the structure perfection of Czochralski-grown $(\mathrm{Cz})$ and float-zone (FZ) silicon crystals.
\end{abstract}

PACS numbers: 81.40.-z, 61.72.Dd

\section{Introduction}

Nearly perfect dislocation-free silicon crystals in initial state contain usually both microdefects and macrostrains of the crystal lattice [1-3]. The latter ones are caused by inhomogeneous (often stratified) distribution of point defects.

To obtain the reliable information about real defect structures one needs to separate the effects of long range elastic strains and localized deformation fields caused by microdefects on diffraction parameters. It should be noted that the long range deformations are assumed to have characteristic size $l^{*}$ which well exceeds the extinction length $\Lambda$ for a used reflection [4]. But the mentioned value $\Lambda$ exceeds considerably that size $l^{*}$ in the case of microdefects.

The radical change of the diffraction beam intensity behaviour occurs when the microdefects are present in elastically strained crystal [5-7]. Therefore the 
interpretation of experimental data is a rather difficult task in the case when there are combined distortions of structure.

Since a strict theory of X-ray dynamical diffraction by a crystal with combined structure distortions has not been developed yet, the search of phenomenological ways for solving the mentioned problem is of interest.

The new X-ray acoustic method of structure diagnostics of slightly imperfect crystals has been developed recently [8-10]. This method is based on the selective suppression of coherent part of total reflectivity by weak ultrasound vibrations. It enables to measure $\Lambda$ of slightly imperfect crystals with high accuracy as well as to reveal the presence of weak macrodeformations in a sample and to determine their level $\varepsilon$. According to [8-10]

$$
\begin{aligned}
& \Lambda=\frac{c_{\mathrm{s}}}{\nu_{\mathrm{s}}}\left[1-(\Delta x / 2 t \sin \Theta)^{2}\right]^{-\frac{1}{2}}, \\
& \varepsilon=2 \frac{d}{\Lambda}\left[\left(\Lambda \nu_{\mathrm{s}}^{*} / c_{\mathrm{s}}\right)^{2}-1\right]^{\frac{1}{2}} .
\end{aligned}
$$

Here $c_{\mathrm{s}}$ and $\nu_{\mathrm{s}}$ are the ultrasound velocity and frequency respectively; $t, \theta$ and $d$ stand for a crystal thickness, a Bragg angle, and an interplanar space, respectively; $\nu_{s}^{*}$ is the specific frequency at which the fine structure of an intensity profile $I(x)$ disappears due to movement of the corresponding tie points along branches of Ewald dispersion surfaces of dynamical diffraction [6]. It should be noted that a wave number of ultrasound $k_{s}\left(k_{s}=2 \pi / \lambda_{s}, \lambda_{s}\right.$ is the wavelength) exceeds slightly in this case the gap of the dynamical dispersion surface $\Delta k_{\mathrm{p}}$ for a perfect crystal $\left(\Delta k_{\mathrm{p}}=2 \pi / \Lambda_{\mathrm{p}}\right.$, where $\Lambda_{\mathrm{p}}$ is the extinction length for an ideal crystal medium).

The extinction length is known $[2,6]$ to increase due to microdefects formation in a crystal as

$$
\Lambda=\Lambda_{\mathrm{p}} \mathrm{e}^{L} \text {. }
$$

On the other hand, the extinction length $\Lambda$ decreases if there are long range deformations in a crystal [4]. This can entail some errors when one calculates $L$ from experimental $\Lambda$ value by means of (3). In present paper we have suggested some improvement of the known procedure [8-10] by taking into account the effect of weak macrodeformations on $\Lambda$.

\section{Methods}

The suggested approach is based on the analysis of the frequency dependencies for distance between mentioned minima $\Delta x\left(\nu_{\mathrm{s}}\right)$ for two selected reflections. The values of extinction lengths $\Lambda$ may be calculated from $\Delta x\left(\nu_{\mathrm{s}}\right)$ rather exactly [9]. It should be noted that original measurements of dependencies $\Delta x\left(\nu_{\mathrm{s}}\right)$ for perfect crystals were performed in [11]. The comparison of the $\Lambda$ and $\Lambda_{\mathrm{p}}$ values enables to identify the predominate type of structure disturbances for a crystal under investigation $\left(\Lambda>\Lambda_{\mathrm{p}}\right.$ and $\Lambda<\Lambda_{\mathrm{p}}$ in the cases of localized or distributed deformations, respectively $[4,12])$.

If microdefects are dominant type of crystal imperfections $(\Lambda>\Lambda)$ one may estimate the static Debye-Waller factor $\mathrm{e}^{-L}$ value by formula (3). Besides, the additional analysis of integral reflectivity $R_{\mathrm{i}}$ values allows us also to calculate the characteristic size and concentration of microdefects. 
As it will be shown further, a similar approach makes it possible also to estimate $L$ and the period of main long range deformation $\lambda_{m}$ for a sample containing both microdefects and macrodeformations. The corresponding calculations in the framework of perturbation theory of $\mathrm{X}$-ray scattering give the following value of the extinction length for a medium with combined distortions:

$$
\Lambda=\Lambda_{\mathrm{p}} \mathrm{e}^{L} \alpha(\varepsilon),
$$

where $\alpha(\varepsilon)$ is the parameter describing decrease in $\Lambda$ due to long range deformations. Following Ref. [4] one may write the expression for this parameter in the case of static periodical deformation

$$
\alpha=\left\{1-\frac{\left(\Lambda_{\mathrm{p}} / \lambda_{\mathrm{m}}\right)^{2}(H W)^{2}}{4\left[1-\left(\Lambda_{\mathrm{p}} / \lambda_{\mathrm{m}}\right)^{2}\right]}\right\} .
$$

Here $H$ is the reciprocal lattice vector and $W=\varepsilon \lambda / \pi \sqrt{2}$ is the static displacement amplitude.

It is evident that $L$ and $\lambda_{\mathrm{m}}$ values may be estimated by means of formulas (4) and (5) using experimental values of $\varepsilon$ as well as $\Lambda$ obtained for two selected reflections. This way of numerical calculations was applied in the present study.

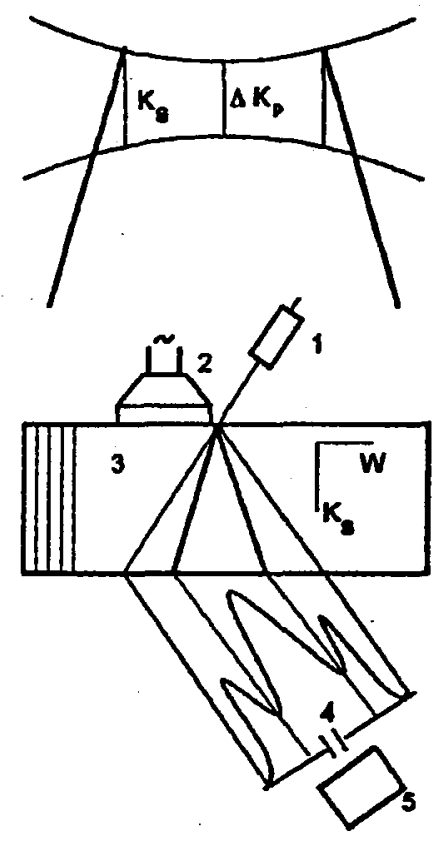

Fig. 1. The scheme of measuring of the interminimum distances $\Delta x\left(\nu_{\mathrm{s}}\right)$ under the X-ray. acoustic resonance conditions. 1 - slits, 2 - transducer, 3 - sample, 4 - detector slit, 5 - detector.

The $\Delta x\left(\nu_{\mathrm{s}}\right)$ values were measured similarly to $[8,9]$ from the intensity profiles of diffracted beams $I(x)$ (Fig. 1) for 220 and 440 reflections of the characteristic Mo $K_{\alpha_{1}}$ radiation (Fig. 2 and Fig. 3 respectively). To estimate the dependencies 


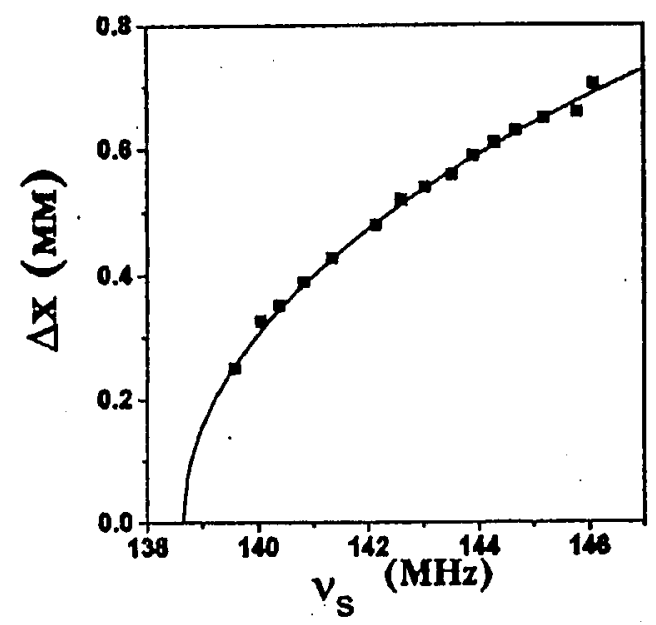

Fig. 2. The $\Delta x\left(\nu_{s}\right)$ dependencies for 220 reflection, Mo $K_{\alpha_{1}}$ radiation. Solid squares correspond to experimental data (specimen 2) and solid line stands for theoretical dependence for a perfect crystal.

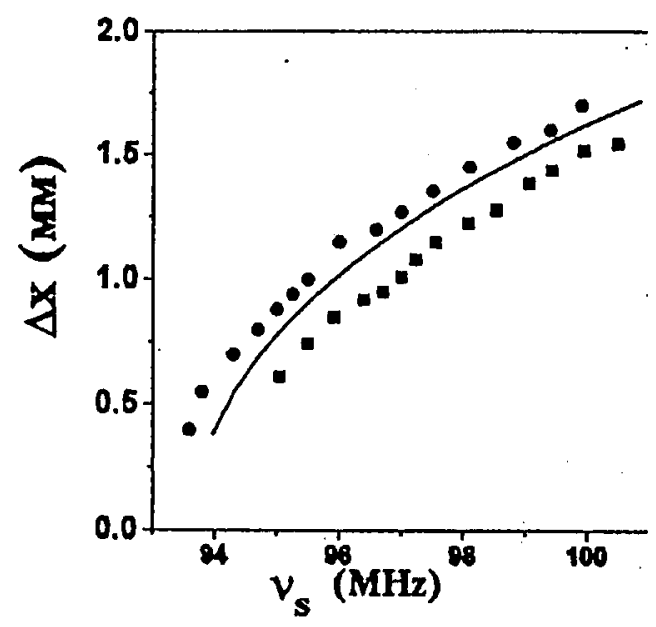

Fig. 3. The $\Delta x\left(\nu_{\mathrm{s}}\right)$ dependencies for 440 reflection, Mo $K_{\alpha_{1}}$ radiation. Solid circles and squares correspond to experimental data (specimen 1 and 2 respectively) and solid line relates to the theoretical curve for a perfect crystal.

$\Delta x\left(\nu_{\mathrm{s}}\right)$ for a perfect crystal we used the structure factor values given in [13]. The $\Lambda$ values were calculated in each case by least-squares fitting of the function (1) to experimental series $\Delta x\left(\nu_{\mathrm{s}}\right)$. The level of $\varepsilon$ was derived from $\nu^{*}$ according to the expression (2). 
The samples under investigation $(t=6 \mathrm{~mm})$ were cut out of a dislocation-free FZ Si ingot (specimen 1) and of $\mathrm{Cz}$ Si one (specimen 2). The oxygen concentration in $\mathrm{Cz} \mathrm{Si}$ was $1.15 \times 10^{24}$ atom. $\mathrm{m}^{-3}$, which was measured by means of infrared spectroscopy. The oxygen content in FZ Si was about two orders lower $\left(10^{22}\right.$ atoms $\left.\cdot \mathrm{m}^{-3}\right)$. The sample surfaces $\{111\}$ were normal to the growth axis. Both lateral and longitudinal stratified patterns were revealed in $\mathrm{Cz}$ Si by means of X-ray topography. The stripe period ranges about 150 to $200 \mu \mathrm{m}$. Defects such as clusters were observed in FZ Si by means of the etch pits counting procedure. The concentration of rather big (mean size of etch pits was about $10 \mu \mathrm{m}$ ) clusters reached $10^{11} \mathrm{~m}^{-3}$ according to that method.

The structure perfection of the crystals under investigation was additionally examined by means of $R_{\mathrm{i}}$ measurements both in the Bragg and the Laue geometries.

Transverse ultrasonic vibrations with wave vector being perpendicular to both the sample surfaces and the diffraction vector were generated by the $\mathrm{LiNbO}_{3}$ piezoelectric transducer. The ultrasound polarization vector was directed normally to the reflecting planes (Fig. 1).

\section{Results and discussion}

The experimental dependencies $\Delta x\left(\nu_{\mathrm{s}}\right)$ observed in specimen 1 proved to be typical of crystals with dominant short range deformations $\left(\Delta x>\Delta x_{p}, \Delta x_{p}\right.$ refers to a perfect sample). In this case the values of $\Lambda$ noticeably exceed $\Lambda_{\mathrm{p}}$, especially for 440 reflection (see Table). The measurements of $\nu_{\mathrm{s}}^{*}$ values for this sample show that the magnitude of $\varepsilon$ is rather low and does not exceed the $10^{-7}$ level.

TABLE

The values of the integral characteristics of structure perfection of the samples under investigation $\left(\Lambda_{\mathrm{p}}(220)=\right.$ $\left.36.50 \mu \mathrm{m}, \Lambda_{\mathrm{p}}(440)=53.99 \mu \mathrm{m}\right)$.

\begin{tabular}{c|c|c|c|c}
\hline \hline Sample number & \multicolumn{2}{|c|}{1} & \multicolumn{2}{|c}{2} \\
\hline Reflection & 220 & 440 & 220 & 440 \\
$\Lambda[\mu \mathrm{m}]$ & 36.54 & 54.27 & 36.50 & 53.62 \\
$L \cdot 10^{3}$, & & & & \\
proposed method & 1.1 & 4.3 & 1.9 & 7.6 \\
$L \cdot 10^{3}$, & & & & \\
Bragg diffraction & 1.1 & 3.5 & - & - \\
$R[\mu \mathrm{m}]$ & 1.1 & 1.1 & - & - \\
$N\left[\mathrm{~m}^{-3}\right]$ & $2.4 \times 10^{11}$ & $3.4 \times 10^{11}$ & - & -
\end{tabular}


Therefore, the static Debye-Waller factor can be correctly estimated for specimen 1 by means of formula (3). It is important to underline that the obtained $L_{440}$ value (Table) is four times higher than $L_{220}$ (increased proportionally to $H^{2}$ ). Such kind of the $L(H)$ dependence corresponds to the cluster-like defects [14].

The $L$ values were also derived from $R_{\mathrm{i}}$ magnitudes measured in the Bragg case of diffraction by numerical solving of the equation [15]:

$$
R_{\mathrm{i}}=R_{\mathrm{i}}^{p} \mathrm{e}^{-L}+R_{\mathrm{i}}^{k}\left(1-\mathrm{e}^{-2 L}\right),
$$

where $R_{\mathrm{i}}^{p}, R_{\mathrm{i}}^{k}$ stand respectively for the integral reflectivities of a perfect and ideally-mosaic crystals. One can see from Table that the results obtained by two independent procedures are in satisfactory agreement which confirms the reability of the information about the degree of the structure perfection of specimen 1 .

If the value of $L$ is known one may determine the magnitude of the second integral structure pararneter $\mu_{\mathrm{d}}$, which describes additional X-ray energy losses due to diffuse scattering by defects, from the $R_{\mathrm{i}}$ value measured in Laue case of diffraction [16]. The characteristic size $R$ and the concentration $N$ of microdefects were estimated from the values of $L$ and $\mu_{\mathrm{d}}$, using the following expressions [17]:

$$
\mu=8 \pi^{2}(\cos \Theta)^{2} L R / \Lambda^{2}, \quad L=8 N R^{9 / 2}(\beta H)^{2},
$$

where $\beta \approx 0.01$. As it follows from Table, a good correspondence was found between two groups of these data obtained for 220 and 440 reflections. Moreover, the satisfactory correlation of these data with the results obtained by the mentioned above metallographic method takes place.

Thus, the suggested approach makes it possible to obtain rather full information about the structure perfection integral characteristics of FZ Si, which contains mainly the short range deformations.

The character of the experimental dependence $\Delta x\left(\nu_{\mathrm{s}}\right)$ for specimen 2 appeared to be different from that for specimen 1 . In this case the measured and calculated dependencies $\Delta x\left(\nu_{\mathrm{s}}\right)$ for 220 reflection almost coincide. $A \mathrm{~s}$ for 440 reflection, the experimental frequency dependence of the $\Delta x$ corresponded to the case of the distributed structure imperfections domination $\left(\Lambda<\Lambda_{\mathrm{p}}\right)$. The $R_{\mathrm{i}}$ values obtained for this specimen in the Laue case of diffraction were about $20 \%$ smaller than those for a perfect crystal both for 220 and 440 reflections. Therefore, one may conclude that macrodeformations determine mainly diffraction processes for 440 reflection while the effects of macrodeformations and microstrains on the $\Lambda$ values are aproximately equal for 220 reflection.

The macrodeformation value $\varepsilon$ was calculated in specimen 2 according to expression (2) by means of the $\nu_{\mathrm{s}}^{*}$ measuring. The magnitude of $\varepsilon\left(3 \times 10^{-7}\right)$ was found to be close to known literature data $[3,10]$. It should be noted that the alternative elastic macrodeformation of crystallographic planes $\{110\}$ was measured in this case.

The system of equations like (4) and (5) was solved numerically for both reflections using dependencies $L(I I)$ known for microdefects [14] $\left(L \sim H^{3 / 2}\right.$ for dislocation loops and $L \sim H^{2}$ for clusters). In the first case the system had no roots. But in the latter one we have obtained the following results: $L_{220}=1.9 \times 10^{-3}$, $L=7.6 \times 10^{-3} ; \lambda=196 \mu \mathrm{m}$. These results happened to be in satisfactory agreement with known literature data [1-3, 6-10]. 
It should be noted that the used approach does not have the potential for unequivocal direct determination of the type of structure defects. Moreover, it can be applied only for investigation of nearly perfect single crystals $\left(L<10^{-2}, \varepsilon \ll \omega\right.$, $\omega$ is an angular width of dynamical diffraction maximum).

\section{Conclusions}

The comparison of experimental data for the extinction lengths of real $(\Lambda)$ and perfect $\left(\Lambda_{\mathrm{p}}\right)$ crystals as well as additional measurements of integral reflectivities allows to get useful information about the structure of slightly imperfect crystals. The method makes it possible to identify the dominant type of structure disturbances and to determine the integral characteristics of microdefects $(L, R, N)$ as well as of macrodeformations $\left(\varepsilon, \lambda_{\mathrm{m}}\right)$.

\section{Acknowledyments}

This work was partially supported by the Ukrainian Committee for Scientific Research and Technology.

\section{References}

[1] S. Iida, H. Sigiyama, Y. Sugita, II. Kawata, Jpn. J. Appl. Phys. 27, 1081 (1988).

[2] S.N. Voronkov, F.N. Chukhovṣkii, P.V. Lomovtsev, S.P. Darbinyan, Plıys. Status Solidi A 134, 301 (1992).

[3] I.R. Entin, I.A. Smirnova, Acta Crystallogr. A 45, 577 (1988).

[4] I.A. Smirnova, I.R. Entin, Phys. Stalus Solidi B 147, 459 (1988).

[5] L.I. Datsenko, V.I. Khrupa, E.N. Kislovskii, Phys. Status Solidi A 68, 399 (1981).

[6] L.I. Datsenko, V.B. Molodkin, M.E. Osinovskii, Dynamicheskoe rasseyanie rentgenovskilh luchey realnymi kristallami, Naukova Dumka, Kiev 1988, p. 198.

[7] D.O. Grigoryev, L.I. Datsenko, V.F. Machulin, V.B. Molodkin, V.I. Khrupa, Metallofizika 15, 23 (1993).

[8] V.I. Klirupa, I.R. Entin, Fiz. Tverd. Tela 33, 1821 (1992).

[9] I.R. Entin, V.I. Khhrupa, O.V. Petrosyan, Phys. Stalus Solidi A 127, 321 (1992).

[10] D.O. Grigoryev, V.F. Machylin, V.I. Khirupa, Zavodskaya Laboratoriya 50, 32 (1993).

[11] I.R. Entin, Phys. Status Solidi B 90, 575 (1978).

[12] L.I. Datsenko, D.O. Grigoriev, A.V. Briginets, V.F. Machulin, V.I. Khrupa, Kristallografiya 39, 61 (1994).

[13] T. Saka, N. Kato, Acla Crystallogr. A 42, 469 (1986).

[14] P. Dederichs, Solid State Phys. 27, 135 (1972).

[15] P.H. Dederichs, Phys. Rev. B 4, 1041 (1971).

[16] L.I. Datsenko, V.I. Khrupa, V.F. Machulin, V.B. Molodkin, Metallofizika 14, 55 (1992).

[17] I.R. Entin, V.I. Khrupa, L.I. Datsenko, J. Appl. Crystallogr. 23, 355 (1990). 